\title{
Technical Note \\ Long Term Indian Ocean Dipole (IOD) Index Prediction Used Deep Learning by convLSTM
}

\author{
Chen $\mathrm{Li}^{1}{ }^{1}$, , Yuan Feng ${ }^{1} * \mathbb{C}^{\mathbb{C}}$, Tianying Sun ${ }^{1}$ and Xingzhi Zhang ${ }^{2}$ \\ 1 College of Information Science and Engineering, Ocean University of China, Qingdao 266100, China; \\ lichen8668@stu.ouc.edu.cn (C.L.); suntianying@stu.ouc.edu.cn (T.S.) \\ 2 Key Laboratory of Physical Oceanography, Institute for Advanced Ocean Studies, Ocean University of China, \\ Qingdao 266100, China; zhangxingzhi@ouc.edu.cn \\ * Correspondence: fengyuan@ouc.edu.cn
}

check for updates

Citation: Li, C.; Feng, Y.; Sun, T.; Zhang, $X$. Long Term Indian Ocean Dipole (IOD) Index Prediction Used Deep Learning by convLSTM. Remote Sens. 2022, 14, 523. https://doi.org/ $10.3390 /$ rs 14030523

Academic Editors: Yunjun Yao, Gad Levy, Xiaotong Zhang, Kun Jia and Ayad M. Fadhil Al-Quraishi

Received: 23 December 2021

Accepted: 19 January 2022

Published: 22 January 2022

Publisher's Note: MDPI stays neutral with regard to jurisdictional claims in published maps and institutional affiliations.

Copyright: (C) 2022 by the authors. Licensee MDPI, Basel, Switzerland. This article is an open access article distributed under the terms and conditions of the Creative Commons Attribution (CC BY) license (https:// creativecommons.org/licenses/by/ $4.0 /)$.

\begin{abstract}
Indian Ocean Dipole (IOD) is a large-scale physical ocean phenomenon in the Indian Ocean that plays an important role in predicting the El Nino Southern Oscillation in the tropical Pacific. Predicting the occurrence of IOD is of great significance to the study of climate change and other marine phenomena. Generally, the IOD index is calculated to judge whether the IOD occurs. In this paper, a convolutional LSTM (convLSTM) neural network is used to build the deep learning model to predict the sea surface temperature in the next seven months and calculate the IOD index. Through the analysis of marine atmospheric data with complex temporal and spatial relationships, the wind field signal knowledge of the physical ocean is introduced to predict IOD phenomenon by combining the prior knowledge of the physical ocean and deep learning. The experimental results show that the average correlation of IOD index time series to the true IOD index time series is $82.87 \%$ from 2015 to 2018, seven months ahead for IOD prediction. IOD manifests as sea surface temperature (SST) anomaly changes, and this thesis verifies that the wind field signal information has a positive impact on the prediction of IOD changes. Moreover, the convLSTM can predict this anomaly better. The IOD index line graph can generally fit the real IOD index variation trend, which has a profound impact on the study of the IOD phenomenon.
\end{abstract}

Keywords: Indian Ocean Dipole (IOD); long term prediction; sea surface temperature (SST); convLSTM; deep learning

\section{Introduction}

IOD is one of the important systems affecting climate anomalies in Asia. Saji et al. [1] proposed the concept of IOD in 1999. Saji defined the IOD index as the mean sea surface temperature in the western Indian Ocean $\left(50^{\circ} \mathrm{E} \sim 70^{\circ} \mathrm{E}, 10^{\circ} \mathrm{S} \sim 10^{\circ} \mathrm{N}\right)$ minus the mean sea surface temperature in the eastern Indian Ocean $\left(90^{\circ} \mathrm{E} \sim 110^{\circ} \mathrm{E}, 10^{\circ} \mathrm{S} \sim 0^{\circ}\right)$. IOD has significant seasonal phase-locking characteristics, which usually start to develop in summer, reaching their peak in autumn, and decaying rapidly in winter [2]. Since 1999, IOD has attracted the attention of scientists at home and abroad.

IOD is of great help in predicting El Nino Southern oscillation [3]. At the same time, IOD can also affect the global climate through large-scale teleconnection [4]. Although IOD only shows the anomaly of SST in the Indian Ocean, the interaction between ocean and atmosphere will have a greater impact, resulting in climate anomalies in areas around the Indian Ocean, Central South America, southern Africa, Southeast Australia, Northeast Asia, and other regions [5-8]. At the beginning of this century, Guan et al. [9] studied the long-range impact of IOD on the climate of East Asia, which mainly transmitted the abnormal signal of IOD to East Asia in the form of wave train, which led to the abnormal temperature in southern China and Japan. Qiu et al. [10] compared the difference of precipitation in southern China under the positive and negative phases of IOD and found 
that in the development stage of the positive phase of IOD, the anticyclonic circulation in the Bay of Bengal transported water vapor to southern China through the South China Sea, causing an abnormal increase in summer precipitation. Xiao et al. [11] also mentioned the impact of IOD on precipitation in the Yangtze River Basin in China through teleconnection. $\mathrm{Xu}$ et al. [12] also studied the impact of IOD and ENSO on precipitation in southern China. Therefore, accurately predicting the occurrence of IOD can provide support for flood control before the arrival of the summer flood season in China.

At present, many scholars at home and abroad have undertaken research on IOD prediction. Wajsowicz and others [13] used the (NSIPP) coupled model prediction system to test the prospect of IOD prediction one quarter or multiple seasons in advance, which proved the predictability of IOD, but the system has large error. The US national environmental prediction coupling system [14] (NCEP CFS) includes global ocean model, atmospheric 3D circulation model, and a land model. The prediction of the coupling system 2 3 months in advance is more advantageous than 5 6 months in advance. In addition, there are SINTEX-F [15], POAMA [16] models, etc. Bao et al. [17] conducted 24 collaborative re-prediction experiments from 1981 to 2017 using the FGOALS-F2 intra seasonal climate prediction system of the Institute of Atmospheric Physics, Chinese Academy of Sciences. According to the prediction results, the prediction fit degree one month in advance is 0.82 , which decreases with the extension of prediction time, and the prediction fit degree five months in advance is 0.56 . Throughout the research process of IOD prediction, from a simple coupling model to a more complex coupling model, to the initialization strategy of full field assimilation and abnormal field assimilation, the optimal interpolation technology (EnOI) and analytical incremental update technology (IAU) [18] are introduced, and finally the stochastic dynamic (SDM) model using seasonal modulation and ENSO forcing [19]. Their prediction time and accuracy cannot meet the needs of experimental research. These methods rely on manual screening and integration, and it is difficult to extract complex data relationships from huge data. With the increasing size of marine data, new methods are needed to digest these data, and the current in-depth learning methods in the field of artificial intelligence respond to this. Ham et al. [20] used CNN to predict enso3.4 index, which could be predicted two years in advance at most, and the correlation decreases with the prediction time became longer. CNN is compared with other methods, and the correlation predicted four months in advance is $75 \sim 85 \%$, while the correlation predicted seven months in advance is 60 75\%. The latest research on IOD is Sarkar's paper published in 2021. Sarkar et al. [21] used 144 different datasets to predict the IOD index for one month and selected the datasets with the best effect to show it. Although Sarkar et al. used LSTM, they only used LSTM for feature extraction. For the regression of IOD index, random forest algorithm (machine learning) is actually used, which is essentially different from our study. In our paper, we only used deep learning.

Artificial intelligence is widely used in image, voice, automatic driving, network security, and so on [22-26], but it is still not closely combined with oceanography, which is the focus of attention in the field of oceanography at home and abroad. Dong [27] et al. used LSTM neural network to predict SST in the next day, three days, one week, and one month, respectively. The experimental results show that LSTM is better than traditional machine learning models (SVR and MLPR) for this time series problem. Nitish Srivastava et al. applied LSTM to video analysis and used historical video sequences for prediction. The model effectively reduced the loss of reconstructed sequences and improved the classification accuracy. In 2012, the Alex net network model built by Alex Krizhevsky et al. [28] significantly reduced the misjudgment rate of image classification by $10.9 \%$ to $15.3 \%$. Convolutional neural network models, such as Google net, Caffe net, and VGG, constantly refresh the accuracy limit of deep learning in image recognition. According to previous studies, we draw the following conclusions: LSTM is widely used in timing problems, while CNN is good at mining spatial features, and the combination of the two can consider timing information based on effectively mining spatial features. 
The purpose of this paper is to predict the sea surface temperature from July to December every year and calculate the IOD index. Based on the knowledge of physical oceanography, this paper uses a convLSTM depth neural network that can mine spatial information and temporal information to learn marine information, including sea surface temperature, underwater temperature, current velocity, salinity, sea surface height, and atmospheric information, including temperature, humidity, wind field, and so on. Deep learning can process physical data, but our research not only uses deep learning to process physical data for prediction, but also adds additional physical information concerning the original data to supplement the model, which can enable to explore the impact of different physical factors on the results and improve the accuracy to a certain extent. The wind field signal is used as one of the factors that can cause IOD to affect the model. Before the data are input into the model, the wind field signal is added to each time step for learning, so the model can consider the impact of wind field on IOD events and better predict such anomalies in SST variability. With the neural network's ability to learn space-time problems and the intervention of physical oceanography knowledge, the IOD index predicted in this paper has a high degree of fitting and can fit the change trend of the real IOD index.

\section{Materials and Methods}

\subsection{Materials}

In this paper, the reanalysis dataset was provided by the American Meteorological Environment Prediction Center (NCEP). The spatial resolution is $1^{\circ} \times 1^{\circ}$. The data set has the characteristics of many elements, wide range, and long time. The reanalysis data set provided by NCEP contains monthly data, which can be found at https:/ / psl.noaa.gov / data/gridded/ (accessed on 22 December 2021). The 81 features used in this paper are shown in Table 1.

Table 1. 81 Factors used in the model.

\begin{tabular}{|c|c|c|c|c|c|}
\hline \multicolumn{6}{|c|}{ Parameters of atmosphere } \\
\hline AT 1000hpa & AT 850hpa & AT 500hpa & AT 300hpa & GPH 1000hpa & GPH 850hpa \\
\hline GPH 500hpa & GPH 300hpa & VV 1000hpa & VV 850hpa & VV 500hpa & VV 300hpa \\
\hline RH1000hpa & RH 850hpa & RH 500hpa & RH 300hpa & ZWS 1000hpa & ZWS 850hpa \\
\hline ZWS 500hpa & ZWS 300hpa & MWS 1000hpa & MWS 850hpa & MWS 500hpa & MWS 300hpa \\
\hline \multicolumn{6}{|c|}{ Parameters of subsea } \\
\hline $\mathrm{SS} 5 \mathrm{~m}$ & SS $15 \mathrm{~m}$ & SS $25 \mathrm{~m}$ & SS 35m & $\mathrm{SS} 45 \mathrm{~m}$ & SS $55 \mathrm{~m}$ \\
\hline SS $65 \mathrm{~m}$ & SS 75m & SS 85m & SS 95m & $\mathrm{u} 5 \mathrm{~m}$ & $\mathrm{u} 15 \mathrm{~m}$ \\
\hline $\mathrm{u} 25 \mathrm{~m}$ & $\mathrm{u} 35 \mathrm{~m}$ & $\mathrm{u} 45 \mathrm{~m}$ & $\mathrm{u} 55 \mathrm{~m}$ & $\mathrm{u} 65 \mathrm{~m}$ & $\mathrm{u} 75 \mathrm{~m}$ \\
\hline $\mathrm{u} 85 \mathrm{~m}$ & u $95 \mathrm{~m}$ & $\mathrm{v} 5 \mathrm{~m}$ & v $15 \mathrm{~m}$ & v $25 \mathrm{~m}$ & v $35 \mathrm{~m}$ \\
\hline v $45 \mathrm{~m}$ & v 55m & v $65 \mathrm{~m}$ & v 75m & v $85 \mathrm{~m}$ & v 95m \\
\hline ST 5m & ST $15 \mathrm{~m}$ & ST $25 \mathrm{~m}$ & ST $35 \mathrm{~m}$ & ST $45 \mathrm{~m}$ & ST $55 \mathrm{~m}$ \\
\hline ST $65 \mathrm{~m}$ & ST $75 \mathrm{~m}$ & ST $85 \mathrm{~m}$ & ST $95 \mathrm{~m}$ & & \\
\hline \multicolumn{6}{|c|}{ Parameters of sea surface } \\
\hline SST & sub1_SST & sub2_SST & sub3_SST & sub4_SST & sub5_SST \\
\hline sub6_SST & sub7_SST & sub8_SST & sub9_SST & sub10_SST & sub11_SST \\
\hline sub12_SST & sub13_SST & sub14_SST & sub15_SST & SSH & \\
\hline \multicolumn{6}{|c|}{ Parameters of wind field } \\
\hline sub1_U & sub2_U & sub3_U & sub4_U & sub5_U & sub6_U \\
\hline sub7_U & sub8_U & sub9_U & sub10_U & sub11_U & sub12_U \\
\hline sub13_U & sub14_U & sub15_U & & & \\
\hline
\end{tabular}

This paper considers the Indian Ocean, which is the third largest ocean in the world $\left(30^{\circ} \mathrm{E}-135^{\circ} \mathrm{E}, 30^{\circ} \mathrm{N}-66.5^{\circ} \mathrm{S}\right)$. In this paper, the sea areas with the range of $40^{\circ} \mathrm{E}-110^{\circ} \mathrm{E}$, $-25^{\circ} \mathrm{S}-25^{\circ} \mathrm{N}$ are selected from the reanalysis data set provided by NECP, and the data set is constructed by sliding window using the data from 1980 to 2018 . 
The training set of 2015 prediction model contains the data from 1980 to 2014. The sliding window slides for one year each time. The training data set contained a total of 63,325 pieces of data, also known as sample number. The test set contains data from 2015-2018 (a total of 2533). This paper forecasts the selected sea areas, selects the ocean atmosphere data of ten consecutive years and January to May of the eleventh year, and forecasts the sea surface temperature from June to December of the eleventh year. Subtract the average sea surface temperature of the western region $\left(50^{\circ} \mathrm{E}-70^{\circ} \mathrm{E}, 10^{\circ} \mathrm{S}-10^{\circ} \mathrm{N}\right)$ from the average sea surface temperature of the eastern region $\left(90^{\circ} \mathrm{E}-110^{\circ} \mathrm{E}, 10^{\circ} \mathrm{S}-0^{\circ}\right)$ allows to obtain the IOD index of every month and observe the change of the IOD index through the broken line chart. The broken line chart shows the change of the 7 months of the IOD index.

The monthly ocean atmosphere data in this paper consist of 81 features. The possible factors affecting the sea surface temperature of data points are divided into three parts: atmosphere, sea surface, and underwater parameters. This paper selects the data of atmospheric temperature, geopotential height, vertical velocity, water vapor, east-west wind speed, and north-south wind speed of different heights (1000, 850, 500, 300 HPA), for a total of 24 atmospheric factors. The sea surface parameters include the sea surface height (SSH) of the center point, the sea surface temperature (SST) of the center point, and the sea surface temperature of 15 surrounding points, for a total of 17 . The subsea parameters include the temperature, east-west current, north-south current, and salinity of different sea depths $(5,15,25,35,45,55,65,75,85,95 \mathrm{~m})$ at the central point, for a total of 40 .

This paper introduces the wind field data into the characteristics to explore the impact of wind field data on IOD index. By adding the wind speed of the first 6 months around 15 points to the monthly ocean atmosphere data, a data set with different characteristics is constructed (the data of each month are composed of 96 characteristics). This paper does not only select SST for prediction, but uses the prior knowledge of physical ocean and comprehensively considers various factors affecting SST, focusing on exploring the effect of wind field information on model effects and on the prediction of the IOD phenomena. This also meets the requirements of predicting the IOD index. The IOD phenomenon is a large-scale and long-period marine physical phenomenon in the Indian Ocean basin, which is affected by air sea movement, ocean current, and local wind field, and is related to long-period marine changes. This paper uses more than ten years of complex data to predict sea surface temperature, not only mining the time series relationship of sea surface temperature, but also integrating marine knowledge, mining information from various factors, fusion analysis, and focusing on the impact of wind field on IOD index.

\subsection{Method}

In this paper, the convLSTM method proposed by Shi [29] is used. The traditional FC LSTM does not consider the spatial correlation, but only the temporal correlation. On the contrary, CNN's most important operation is convolution, which can extract spatial features well, but cannot grasp the time sequence information. The antagonistic neural network (GAN) is a generative model which is trained in an antagonistic way. It is not mature enough for time series prediction. Since the dataset we consider is relatively large and contains many data on the ocean atmosphere, convLSTM can better mine spatial correlation based on the problem of time series. In the process of predicting sea surface temperature, in order to better extract the ocean atmosphere features, convLSTM method is used to convolute the ocean atmosphere data at each time step to extract the features. The cell structure of convLSTM is shown in Figure 1.

$$
\begin{gathered}
i_{t}=\sigma\left(W_{x i} * X_{t}+W_{h i} * H_{t-1}+W_{c i}{ }^{\circ} C_{t-1}+b_{i}\right) \\
f_{t}=\sigma\left(W_{x f} * X_{t}+W_{h f} * H_{t-1}+W_{c f}{ }^{\circ} C_{t-1}+b_{f}\right) \\
C_{t}=f_{t}^{\circ} C_{t-1}+i_{t}^{\circ} \tanh \left(W_{x c} * X_{t}+W_{h c} * H_{t-1}+b_{c}\right) \\
O_{t}=\sigma\left(W_{x o} * X_{t}+W_{h o} * H_{t-1}+W_{c o}{ }^{\circ} C_{t}+b_{o}\right)
\end{gathered}
$$




$$
H_{t}=o_{t}{ }^{\circ} \tanh \left(C_{t}\right)
$$

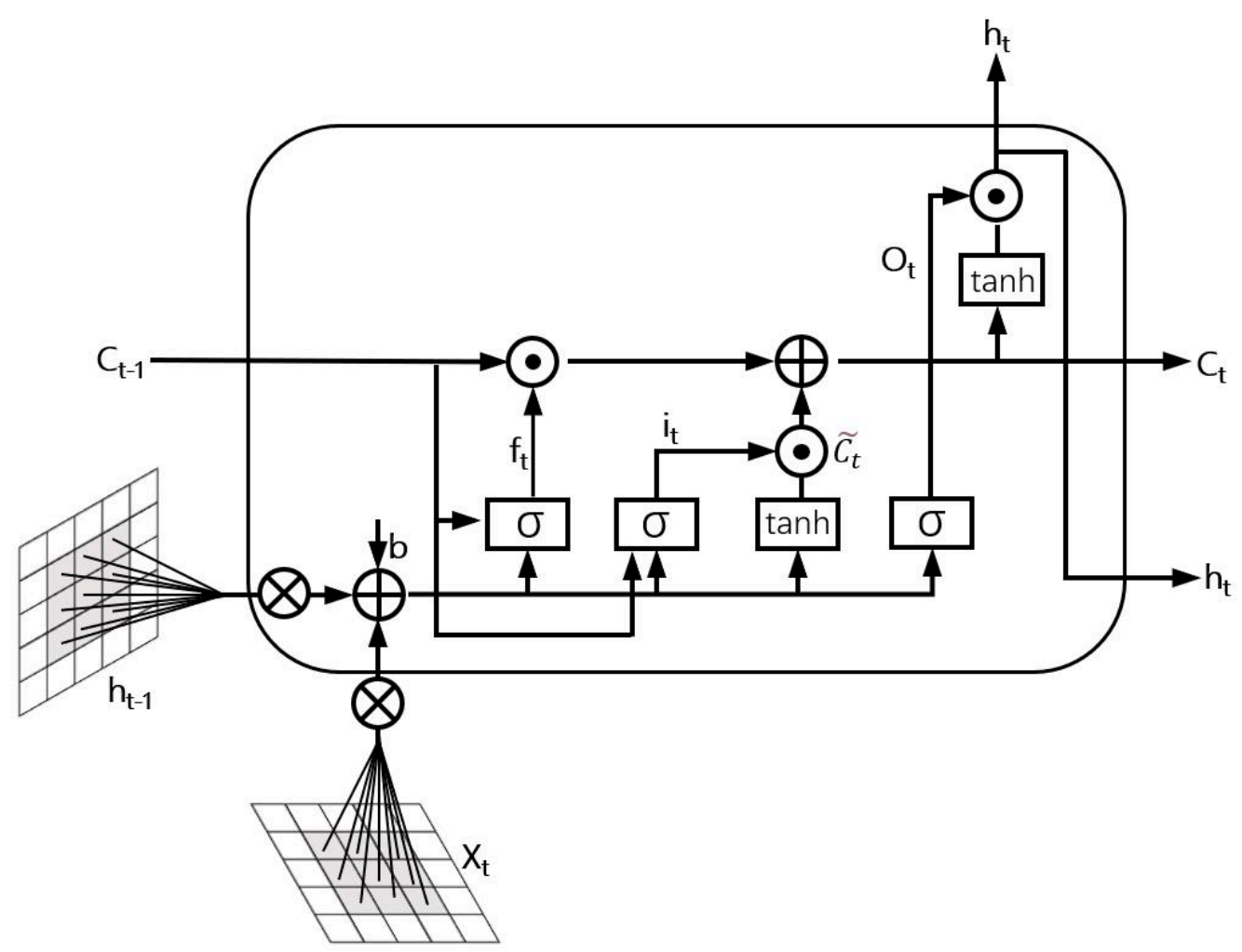

Figure 1. One cell structure of convLSTM.

The convLSTM structure is shown in the figure above, where I, F, C, O, and h represent input gate, forgetting gate, cell state, output gate, and output at a certain time, respectively. Formulae (1)-(5) provide the calculation process of I, F, C, O, and $\mathrm{H}$ respectively, where $\mathrm{W}$ represents the parameter matrix, the subscript is used to distinguish different parameter matrices, $X_{t}$ represents the input at time $t, H_{t-1}$ represents the output at the previous time, $C_{t-1}$ represents the cell state at the previous time, and $\mathrm{b}$ represents the offset, while $\sigma$ indicates that SIGMOD activation function and maps the value to $0-1$. For example, $\mathrm{ft}$ is mapped to a 0-1 matrix, while $f_{t}^{\circ} C_{t-1}$ controls what information needs to be forgotten in the $C_{t-1}$. If the corresponding element in the $\mathrm{ft}$ matrix is 0 , it means that all the information is forgotten. If it is 1 , it means that all the information is retained. Compared with LSTM, where $\mathrm{x}, \mathrm{C}, \mathrm{h}, \mathrm{I}, \mathrm{O}$, and $\mathrm{f}$ become three-dimensional tensors, * represents convolution operation, and ${ }^{\circ}$ represents the multiplication of corresponding elements of the matrix, also known as the Hadamard product. convLSTM uses the three-gate control structure of LSTM, which can forget the unimportant information in history and retain the important information. It performs well on the problem of long-time series and can effectively avoid the problem of RNN gradient disappearance and gradient explosion. The convLSTM method can convolute complex spatial features and has strong memory ability for long-time series, so we chose this method.

The convLSTM network architecture used in this paper is shown in Figure 2. The input data are 25 time steps, where each time step has 405 features, and the SST in the next 7 months is obtained through two convLSTM layers. At the same time, in order to explore the impact of wind field on iod, 75 characteristic wind field data are added to each time step. 


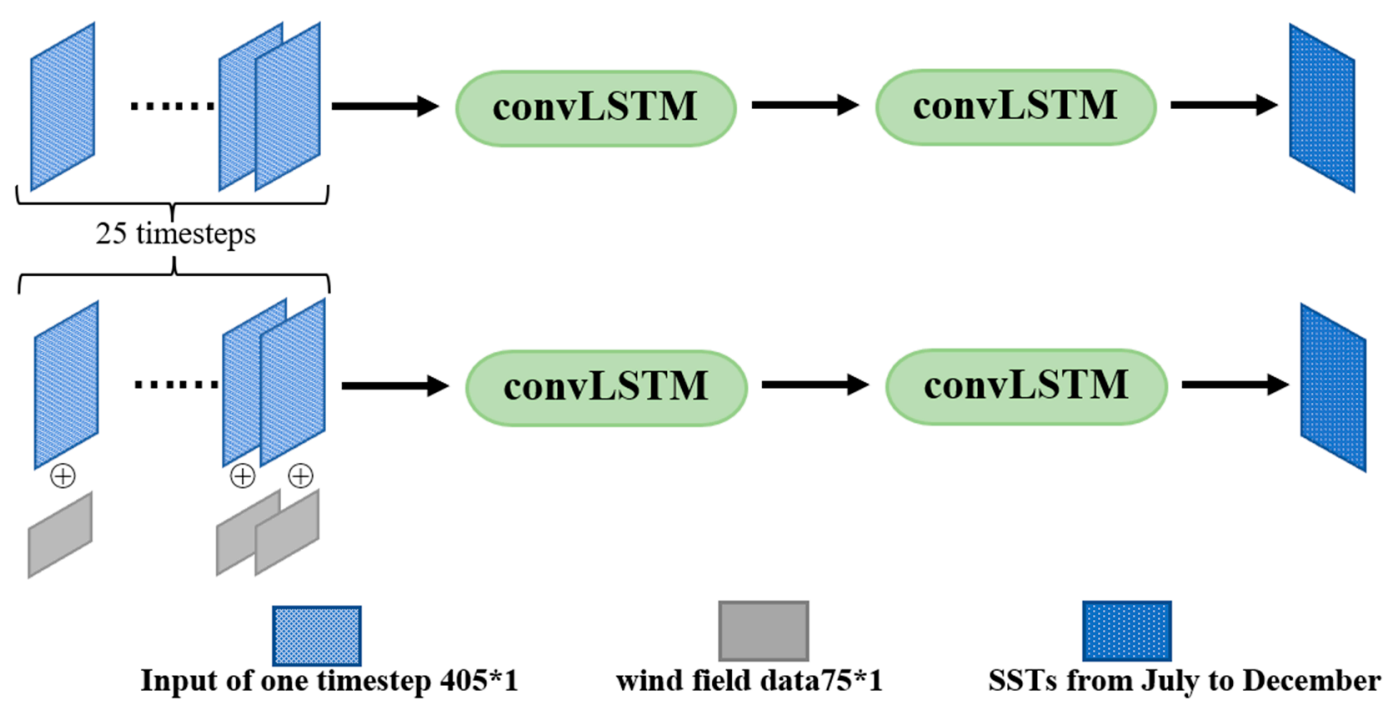

Figure 2. convLSTM structure, including two ConvLSTM2d layers.

The purpose of this paper is to predict the sea surface temperature from July to December every year. The time series is long, and a multi-step prediction model needs to be provided. When designing the model, one month is not selected as a time step. If the number of time steps is set to 125 , gradient explosion will occur due to the long time series. In order to better extract spatial-temporal information and avoid gradient problems, the five-month ocean atmosphere information is regarded as a time step to reduce the number of time steps and can consider data for longer periods of time in one time step. Due to the long time period of IOD, in order to make our training data include a complete period of IOD events, we use the information of ten years of history to predict. In the process of setting convLSTM super parameters, it is found that convLSTM uses one-dimensional convolution to have more advantages for the processing of this long-time series. In this paper, the convolution method in convLSTM is set to one-dimensional convolution by changing the dimension of samples. In the process of the experiment, it was found that due to the complex structure of convLSTM and the excessive number of iterations of the training set, the model parameter matrix will be completely suitable for the training set data but cannot fit the test set. This would lead to over fitting. Considering the actual situation in the process of adjusting parameters, this paper sets the epoch to 100 to make the fitting degree of the model to the training set achieve just good results. The number of model layers is directly related to the prediction effect. When using a CNN model to predict sea surface temperature, the convolution layer of 3-5 layers is generally used, but the model in this paper only uses a two-layer convLSTM layer to predict sea surface temperature. This is because convLSTM is a combination of LSTM and CNN neural networks. convLSTM has strong mining ability for spatiotemporal data. In the experiment, increasing the number of model layers will lead to over fitting. The convLSTM model with two convLSTM layers and one dense layer has the best effect.

In this paper, the model uses Adam as the optimizer and MSE as the loss function. The size of convolution kernel is $(1,3)$. Using two-layer convLSTM $2 \mathrm{~d}$, the number of neurons is set to 42 , each time step returns the output as the input of the next time step, and the convolution filling is set to the same filling. The output of convlstm $2 \mathrm{~d}$ is directly mapped to the 7-month SST we want to predict through a full connection layer to realize multi-step output.

The main advantage of Adam optimizer is its adaptive learning rate and momentum gradient reduction. The main formula is as follows:

$$
m_{t}=\operatorname{beta}_{1} * m_{t-1}+\left(1-\text { beta }_{1}\right) * g
$$




$$
\begin{gathered}
v_{t}=\text { beta }_{2} * v_{t-1}+\left(1-\text { beta }_{2}\right) * g * g \\
\text { variable }=\text { variable }-l r_{t} * m_{t} /\left(\sqrt{v_{t}}+\varepsilon\right)
\end{gathered}
$$

where $m_{t}$ represents the first-order exponential smoothing of the historical gradient and $v_{t}$ represents the first-order exponential smoothing of the square of the historical gradient. Variable represents the gradient update amount at the current time, which is directly proportional to $m_{t}$ and inversely proportional to $v_{t}$. In the process of gradient update, the first-order exponential smoothing can reduce the oscillation during gradient update and obtain a relatively stable gradient update value. The role of $v_{t}$ is to enable Adam to have the ability of adaptive learning rate. When the gradient is stable, it can adaptively reduce the learning rate. Similarly, when the gradient is stable, it can automatically increase the learning rate. The formula for mean square error (MSE) is as follows:

$$
M S E=\frac{\sum_{i=1}^{m}\left(X_{\text {pred }, i}-X_{\text {real }, i}\right)^{2}}{m}
$$

MSE measures the average error of all samples. The smaller the MSE, the better the convergence of the model, the smaller the error, and MSE converges relatively fast, which is suitable for this regression model.

For the prediction of SST, we use root mean square error (RMSE) and accuracy (ACC) as evaluation indicators. RMSE is used to measure the deviation between the observed value and the true value and can reflect the actual situation of the error of the predicted value. The formula is as follows:

$$
\begin{gathered}
\text { RMSE }=\sqrt{\frac{\sum_{i=1}^{m}\left(X_{\text {pred }, i}-X_{\text {real }, i}\right)^{2}}{m}} \\
\text { Accuracy }=1-\frac{\sum_{i=1}^{m}\left(\frac{\left|X_{\text {pred }, i}-X_{\text {real }, i}\right|}{X_{\text {real }, i}}\right)}{m}
\end{gathered}
$$

where $m$ represents the number of sample points. $X($ pred, $i)$ represents the predicted value of the $i$-th sample point, and $X(r e a l, i)$ represents predicted value of the $i$-th sample point.

\section{Results}

This paper takes the ocean atmosphere data of the past 125 months as the training set to predict the sea surface temperature in the next seven months, that is, the sea surface temperature from June to December. For the verification set, $20 \%$ of the training set is used, and the model parameters are adjusted to obtain the optimal model. In the prediction of SST, we use MSE and RMSE as evaluation criteria. For the prediction of IOD index, in order to show the fitting between real IOD and predicted IOD index, we use correlation to show the prediction performance.

\subsection{Comparison of RMSE and ACC}

This paper predicted the sea surface temperature from 2015 to 2018 and forecasts the sea surface temperature from July to December every year respectively. ACC and RMSE are shown in the Table 2. Firstly, the RMSE for the long-term prediction of SST is generally between 0.4 and $0.6^{\circ} \mathrm{C}$, except when the RMSE in individual months exceeds $0.6^{\circ} \mathrm{C}$ (December of 15 years, December of 18 years and September of 2016). From the perspective of RMSE, the prediction error in 2015 and 2016 is greater than that in 2017 and 2018. Before adding wind field data, our model reached a high accuracy. Secondly, the prediction of IOD index in this paper is based on the calculation of sea surface temperature. The prediction of sea surface temperature determines the accuracy of the final IOD index prediction to a certain extent. However, in normal years, there is little difference in RMSE 
between adding a wind farm and not adding a wind farm. It can be seen from the RMSE that the predicted data after adding the wind farm perform better in IOD years.

Table 2. RMSE of the no_wind convLSTM model and convLSTM model with wind field data.

\begin{tabular}{ccccccccc}
\hline \multirow{2}{*}{ RMSE Compare } & \multicolumn{2}{c}{2015} & \multicolumn{2}{c}{$\mathbf{2 0 1 6}$} & \multicolumn{2}{c}{$\mathbf{2 0 1 7}$} & \multicolumn{2}{c}{$\mathbf{2 0 1 8}$} \\
\cline { 2 - 8 } & No_Wind & Wind & No_Wind & Wind & No_Wind & Wind & No_Wind & Wind \\
\hline Jun & 0.564 & 0.448 & 0.541 & 0.513 & 0.322 & 0.398 & 0.414 & 0.456 \\
Jul & 0.501 & 0.481 & 0.646 & 0.639 & 0.467 & 0.566 & 0.444 & 0.456 \\
Aug & 0.513 & 0.531 & 0.497 & 0.476 & 0.487 & 0.528 & 0.534 & 0.560 \\
Sept & 0.471 & 0.464 & 0.642 & 0.625 & 0.498 & 0.488 & 0.431 & 0.435 \\
Oct & 0.590 & 0.528 & 0.583 & 0.578 & 0.431 & 0.546 & 0.447 & 0.472 \\
Nov & 0.508 & 0.496 & 0.505 & 0.527 & 0.435 & 0.485 & 0.519 & 0.446 \\
Dec & 0.698 & 0.639 & 0.507 & 0.515 & 0.595 & 0.575 & 0.597 & 0.606 \\
\hline
\end{tabular}

As shown in Figure 3, the overall accuracy after adding the wind farm is more than $98 \%$, especially in the two IOD years, 2015 and 2016. Although the prediction accuracy of the added wind farm decreased in the normal years, 2016 and 2017, the difference is not large, because the wind field information focuses on guiding the model to predict anomalous changes in SST, i.e., it is better able to predict such anomalies for years in which IOD occurs. Overall, the added wind farm is more stable, and the model for predicting the IOD index proposed in this paper has higher accuracy.

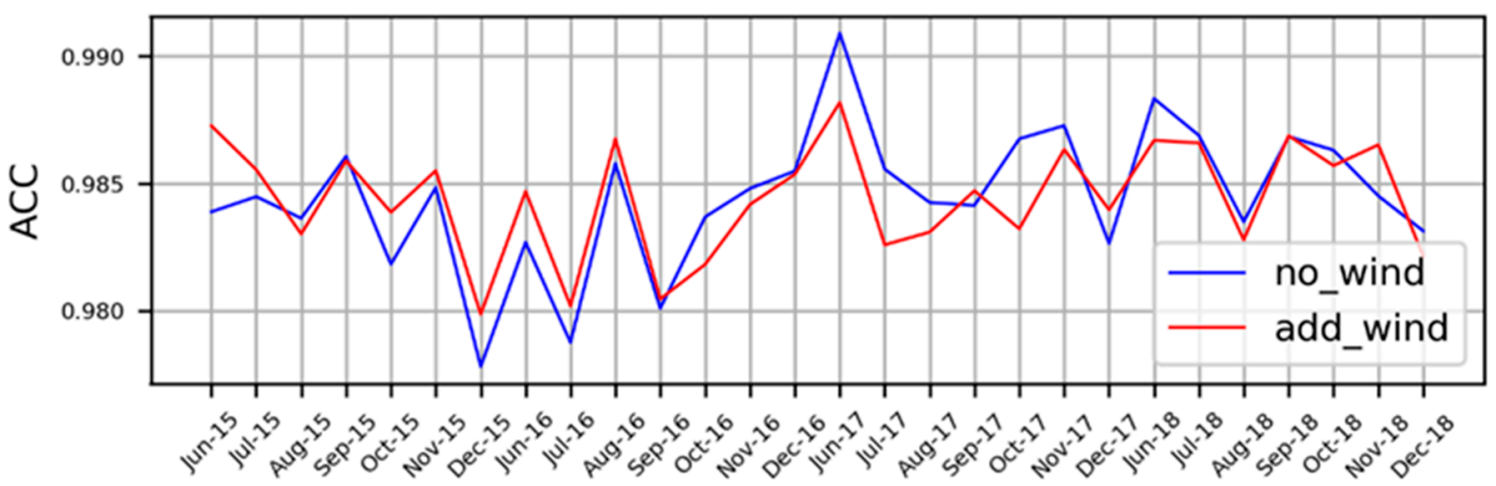

Figure 3. The ACC line chart for each month from 2015 to 2018.The blue line is ACC of convLSTM model without wind and the red line is ACC of convLSTM model with wind field data.

\subsection{Correlation Coefficient between Real IOD Index and Predicted IOD Index}

Taking 2015-2018 as test sets, we use the trained convLSTM method to predict the sea surface temperature and calculate the IOD index by subtracting the average sea surface temperature in the East $\left(50^{\circ} \mathrm{E} \sim 70^{\circ} \mathrm{E}, 10^{\circ} \mathrm{S} \sim 10^{\circ} \mathrm{N}\right)$ from the average sea surface temperature in the $\left(90^{\circ} \mathrm{E}-110^{\circ} \mathrm{E}, 10^{\circ} \mathrm{S}-0^{\circ}\right)$ region. We have made two sets of models. One set is characterized by 81-dimensional ocean and atmosphere data, and the other set is based on 81 dimensional characteristics and the wind field six months ago based on 81 dimensional characteristics in each time step. The IOD index obtained by the two sets of models is shown in the Figure 4. 

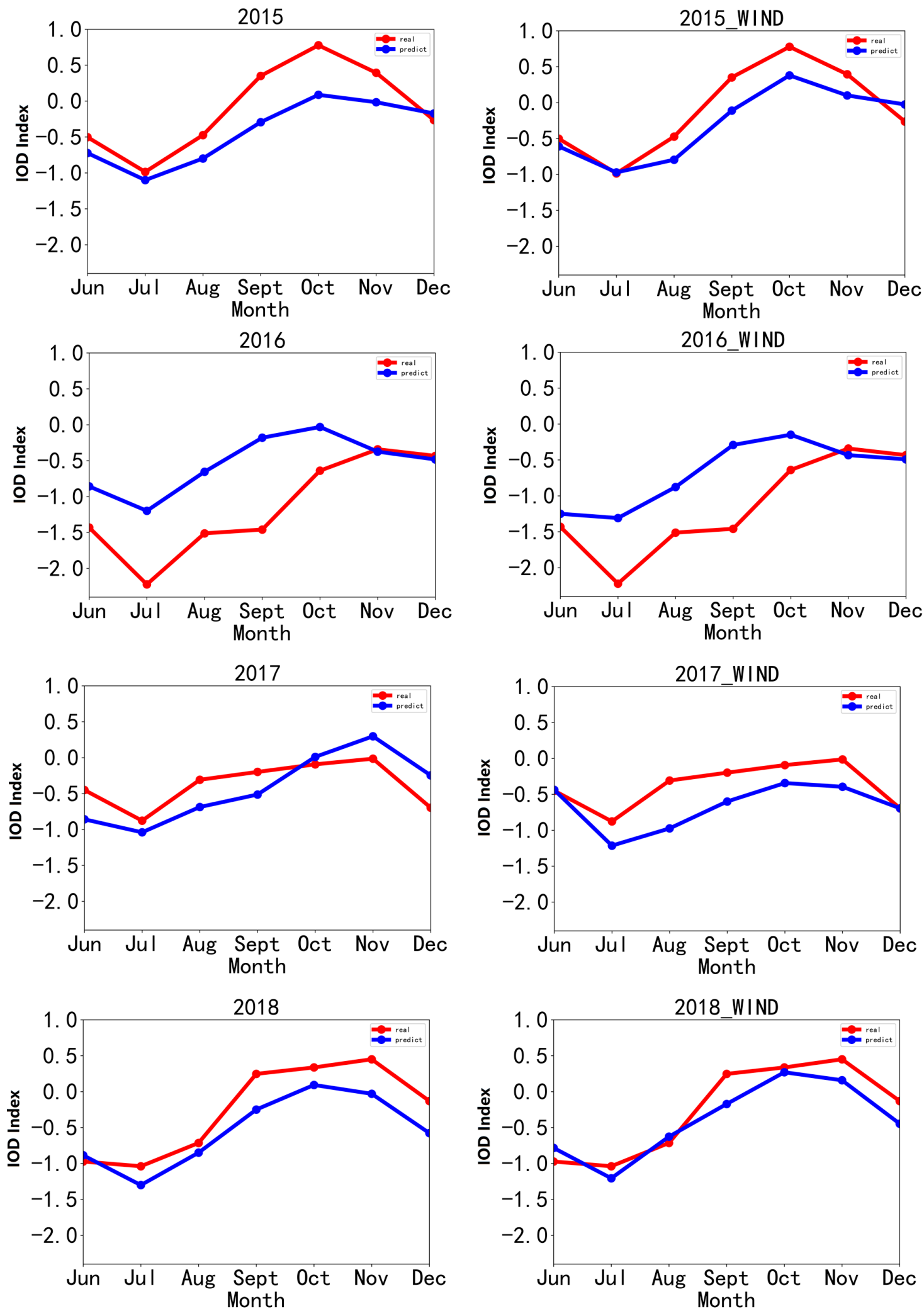

Figure 4. The IOD index of the convLSTM model without wind field data and the index of the convLSTM model with wind field data from 2015 to 2018.

It can be seen from the figure that the IOD index has the characteristics of seasonal change. It starts to rise in summer, reaches the peak in autumn, and decays rapidly in winter. This change is particularly obvious in the super IOD in 2015. The red solid line in the figure 
is the change curve of the real IOD index in 2015. The IOD index peaked in October 2015, close to 1.0. The IOD phenomenon is not only manifested in the obvious increase of IOD index in autumn (called positive IOD phenomenon), but also in the following year. The IOD index will decrease to a trough, which is called negative IOD phenomenon. In 2016, there was a super negative IOD phenomenon. The IOD index showed a downward trend in June and fell to the lowest in July, close to $-2.5^{\circ} \mathrm{C}$. The IOD phenomenon is often a series of positive IOD and negative IOD phenomena. It can be seen from Figure 4 that in 2015-2016, the IOD index series obtained from the predicted SST basically showed the characteristics of seasonal variation of IOD.

In the broken line diagram supplemented with wind field data, the blue line is closer to the red line, and the fitting degree to the real value is higher. The model error of adding wind field data is smaller, which makes the calculated IOD index closer to the real value, which proves that wind field data have a positive impact on IOD index. Overall, the IOD index series calculated by the model prediction in this paper has a high fitting degree to the real IOD change curve. We can see that our prediction line has obvious peaks and valleys, which can determine whether IOD occurs. This is important because, in the future, we can use this model to predict whether IOD will occur this year. Comparing the results of the two sets of experiments in 2017, the error of IOD index in June and December 2017 is eliminated after adding wind field data. Using the model of the original data set, the IOD index rose suddenly in November. After adding the wind field data, this abnormal error in a certain month was solved. This shows that the stability of the model after adding wind field data is higher. It can be seen from the comparison in 2018 that the IOD index is closer to the real IOD index in more months after adding the wind farm. The model in this paper verifies the prior knowledge of marine physics by comparing the two sets of models. Many scholars believe that the local wind field information six months ago affects the IOD phenomenon. It can be seen from the figure that the IOD index calculated by the model with wind field data is closer to the real value, which verifies that there is an important relationship between wind field data and the IOD phenomenon.

In order to show the performance comparison between the convLSTM model and the traditional coupling model, this paper uses the data of the climate prediction system version 2 (CFSv2) of the American National Center for environmental prediction (NCEP). Cfsv2 is a fully coupled model and the model uses the interaction between atmosphere, ocean, land, and sea ice to predict the sea surface temperature in the next nine months. This paper uses the SST predicted by CFSv2 model to calculate the IOD index and then compare the CFSv2 model with the convLSTM model. The IOD index predicted by CFSv2 model is shown in Figure 5.
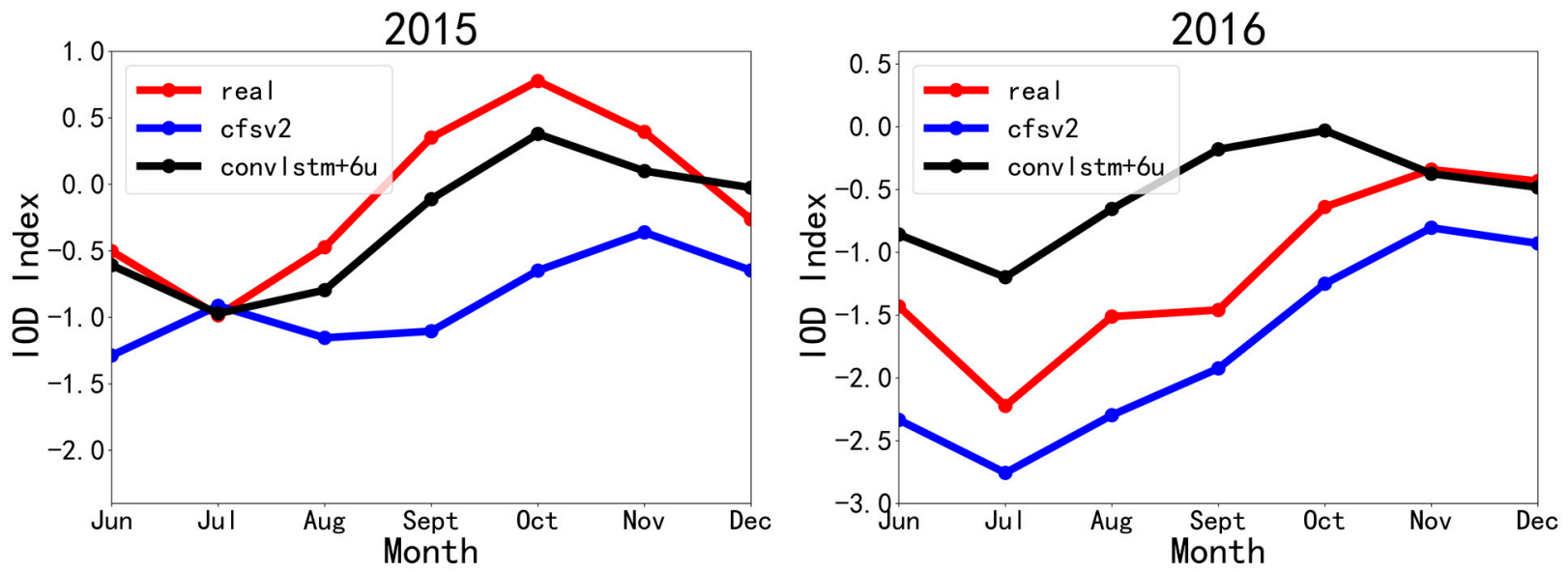

Figure 5. Cont. 

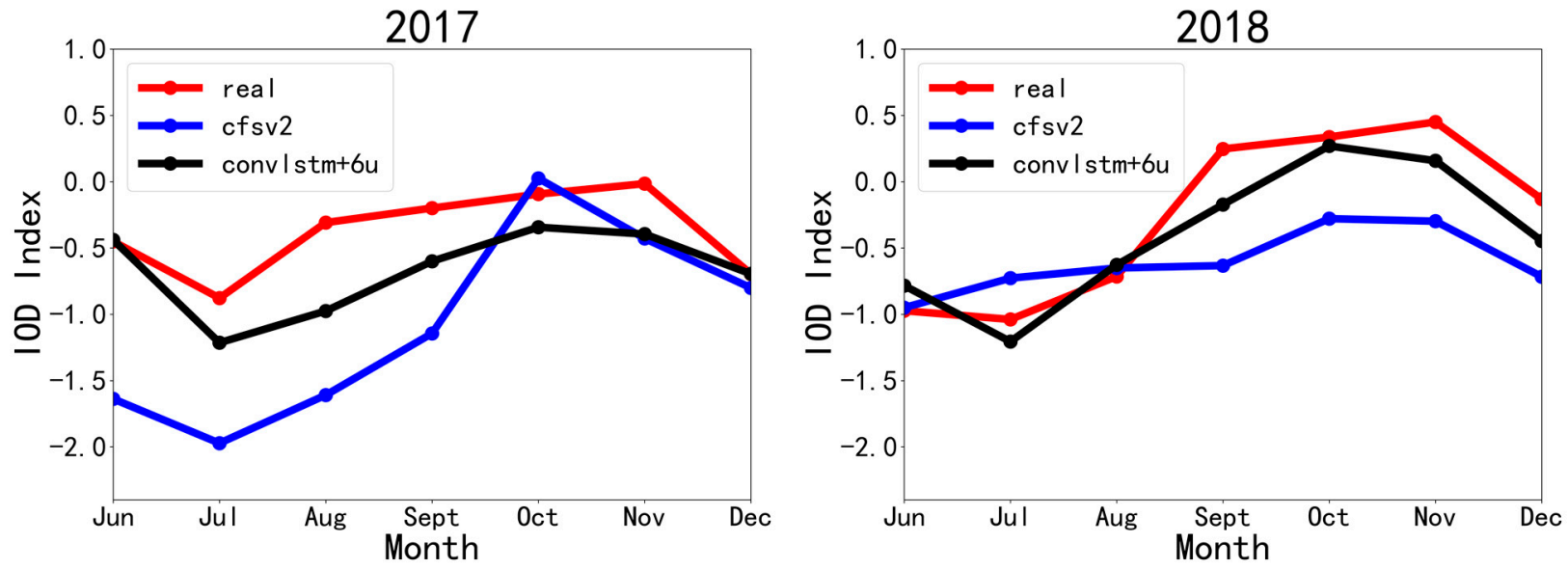

Figure 5. The IOD index of the convLSTM model with wind field data before 6 month and the index of the CFSv2 model from 2015 to 2018.

In Figure 5, we show the IOD index obtained by the CFSv2 model and convLSTM model with wind field information. For the super positive IOD in 2015, the IOD index rose to the peak in October and was close to 0.8 . The CFSv2 model lagged, and the prediction intensity was insufficient. The index of each month was below 0 , and the IOD index rose to the peak in November, while convLSTM was closer to the real value in trend and intensity. For 2016, although the CFSv2 model had some errors, it can better predict the trend of IOD index, while the prediction intensity of the convLSTM model from June to October 2016 is not enough. For the two normal years of 2017 and 2018, convLSTM is closer to the real value in terms of trend and error. On the whole, the convLSTM model with wind information is better.

The correlation coefficient is a statistical index that can reflect the close degree of correlation between variables. The closer the value of the correlation coefficient is to 1 proves that the correlation of the two groups of variables is higher. We calculated the correlation coefficient between the predicted seven-month IOD index and the real IOD index. The higher the correlation coefficient, the closer the predicted IOD index curve is to the change trend of the real value. The correlation coefficient is shown in the Table 3.

Table 3. The correlation of no_wind convLSTM model and wind convLSTM model and CFSv2 model from 2015 to 2018.

\begin{tabular}{cccccc}
\hline Years & $\mathbf{2 0 1 5}$ & $\mathbf{2 0 1 6}$ & $\mathbf{2 0 1 7}$ & $\mathbf{2 0 1 8}$ & avg \\
\hline convLSTM & $91.51 \%$ & $69.50 \%$ & $69.17 \%$ & $95.73 \%$ & $81.48 \%$ \\
\hline convLSTM+6u & $92.24 \%$ & $72.24 \%$ & $72.36 \%$ & $94.52 \%$ & $82.84 \%$ \\
\hline CFSv2 & $49.33 \%$ & $97.67 \%$ & $65.53 \%$ & $80.53 \%$ & $73.27 \%$ \\
\hline
\end{tabular}

It can be seen from the IOD index chart that after adding the wind farm to the original data, the prediction correlation coefficient of IOD index in 2015-2017 has improved, while the average correlation coefficient has also improved. For the super positive IOD events in 2015 , the correlation has increased from $91.51 \%$ to $92.24 \%$. For the super negative IOD event in 2016, the prediction is also closer, and the correlation has increased from $69.50 \%$ to $72.24 \%$. However, the CFSv2 model performed better in 2016. CFSv2 model almost perfectly predicted the real trend of IOD index in 2016, and the correlation reached $97.67 \%$. In terms of correlation coefficient, the correlation coefficient in 2015, 2016, and 2017 increased significantly. The average correlation between 2015 and 2018 increased from $81.48 \%$ to $82.84 \%$. However, our prediction intensity is still not high enough. In the prediction of normal years (2017 and 2018), the fitting degree of IOD index is good. The correlations of the cfsv2 model in 2015, 2017, and 2018 are relatively low, which can not 
well predict super positive IOD events, and the prediction accuracy for normal years is not high enough. On the whole, the convLSTM model with wind field has a higher correlation.

The sea surface temperature predicted by the convLSTM model with wind field information is shown in Figure 6. It can be seen from the real SST map from June to December 2015 that the sea surface temperature of the Indian Ocean is higher in the East and lower in the West in June, and the sea surface temperature in the Western Sea area is higher than that in the eastern sea area in October. The east and west sea areas affected by the IOD phenomenon show a seasonal change trend, which is in line with the law that the IOD index drops to the bottom in summer and reaches the peak in autumn. Overall, the predicted thermodynamic map roughly simulates the distribution trend of real SST in the Indian Ocean. From the real thermodynamic map from June to October, the temperatures in the Arabian Sea, Margiela Bay, and the sea area near the equator $\left(7^{\circ} \mathrm{N} \sim 7^{\circ} \mathrm{N}, 50^{\circ} \mathrm{E} \sim 100^{\circ} \mathrm{E}\right)$ are higher, while the temperatures in other sea areas are lower, and the corresponding predicted SST map also has such characteristics, which shows that the accuracy of the model in this paper is high and the error is small. The predicted thermal distribution also conforms to the law of the IOD phenomenon, and the temperature difference between the western and eastern seas changes obviously. In the prediction chart, the temperature in the eastern sea area in June is slightly higher than that in the west, and with the month gradually entering winter, the Indian Ocean shows an overall cooling trend. In October, the temperature in the western sea area increases as a whole, while the overall sea surface temperature in the eastern sea area decreases.
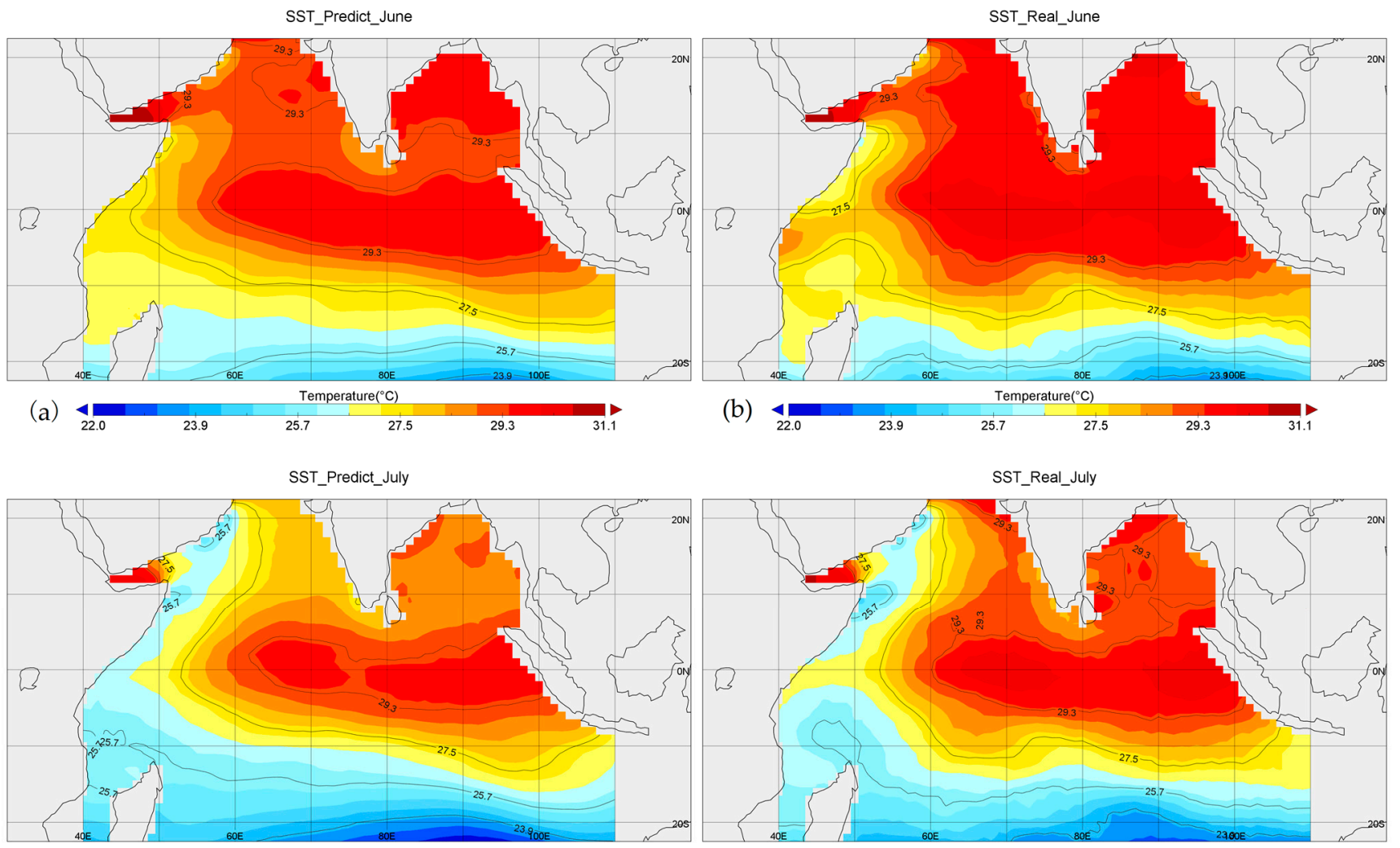

\begin{tabular}{llllll} 
(c) & \multicolumn{5}{c}{ Temperature $\left({ }^{\circ} \mathrm{C}\right)$} \\
\hline 22.0 & 23.9 & 25.7 & 27.5 & 29.3
\end{tabular}

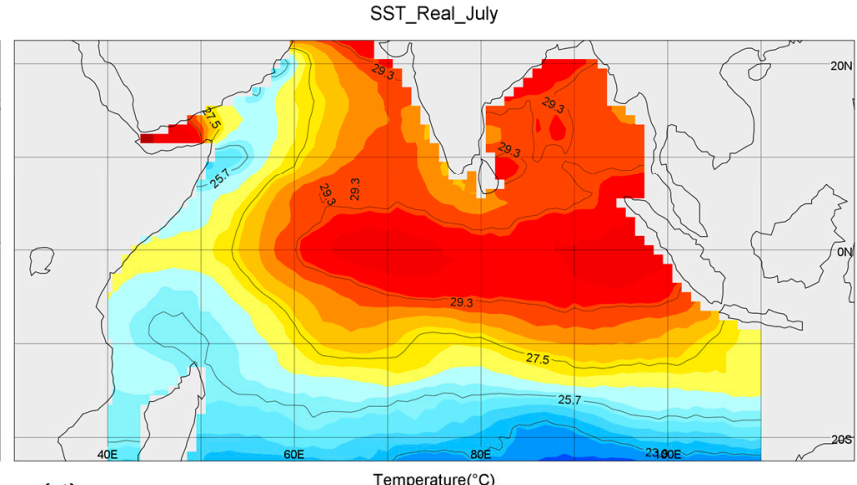

(d)

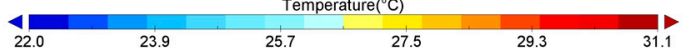

Figure 6. Cont. 


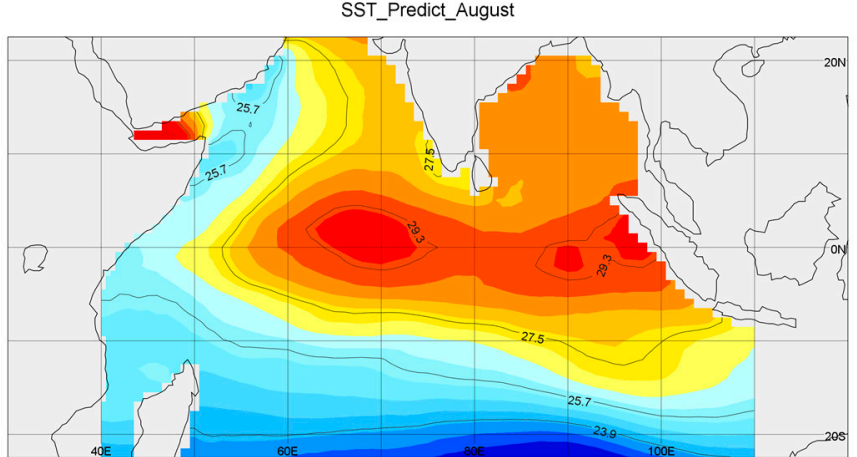

(e) 4

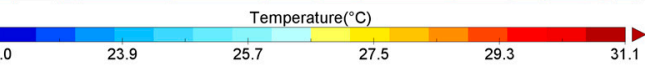

SST_Predict_September

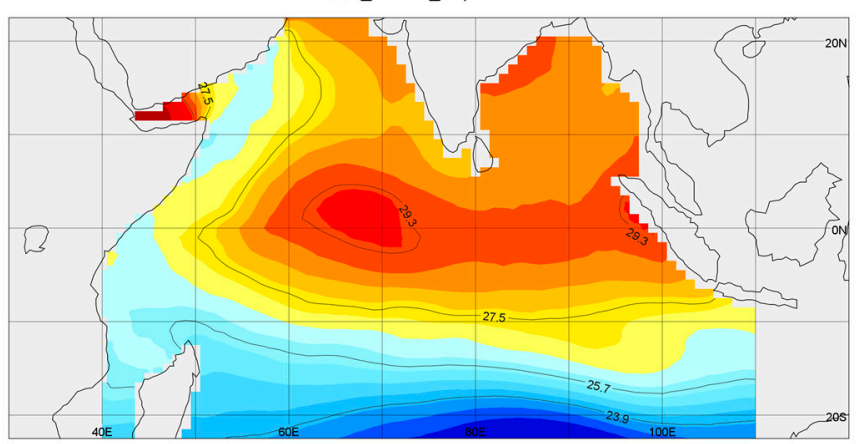

(g) 4

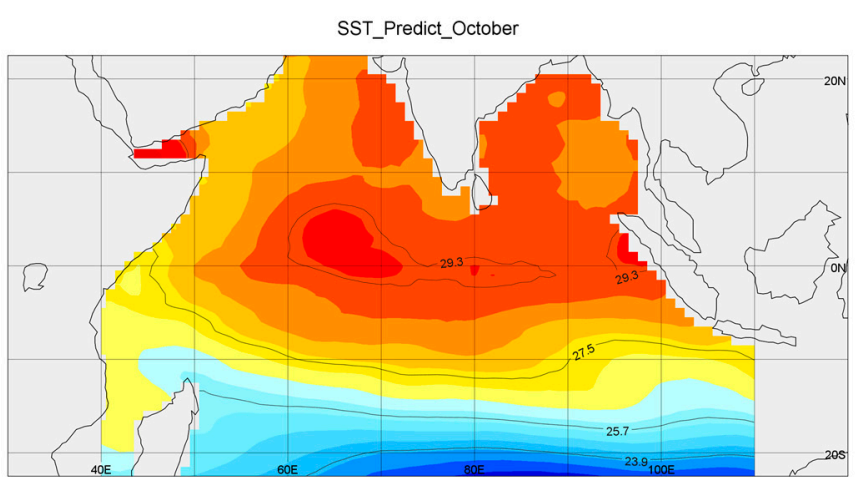

$\begin{array}{lllll}\text { (i) } \begin{array}{r}\text { Temperature }\left({ }^{\circ} \mathrm{C}\right) \\ 22.0\end{array} & 23.9 & 25.7 & 27.5 & 29.3\end{array}$
SST_Real_August

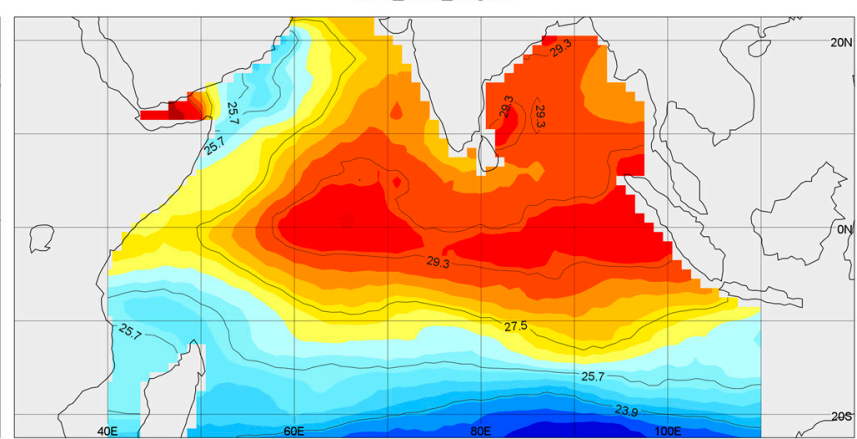

(f)

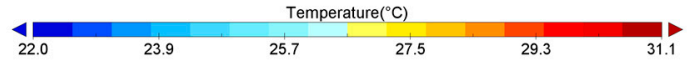

SST_Real_September

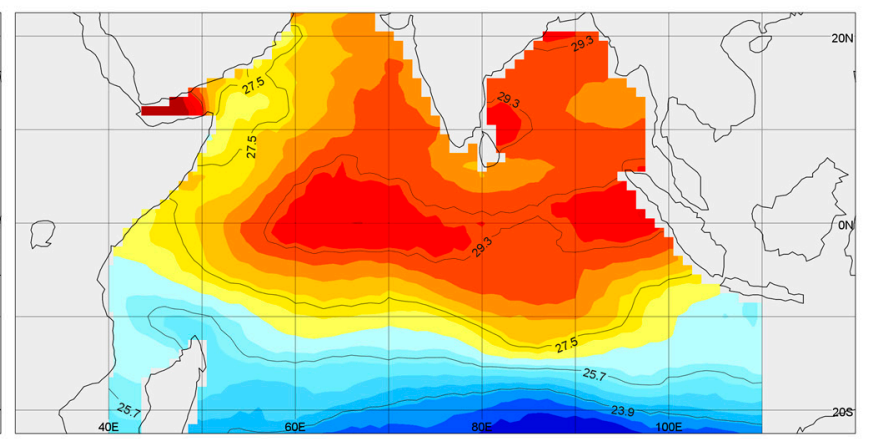

\begin{tabular}{llllll} 
(h) & \multicolumn{5}{c}{ Temperature $\left({ }^{\circ} \mathrm{C}\right)$} \\
\hline 22.0 & 23.9 & 25.7 & 27.5 & 29.3
\end{tabular}

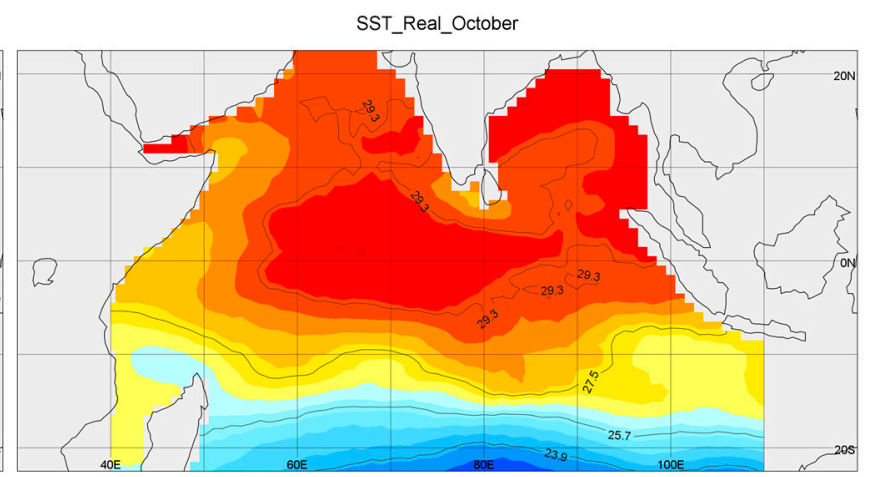

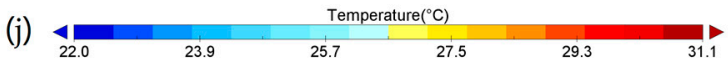

Figure 6. Cont. 


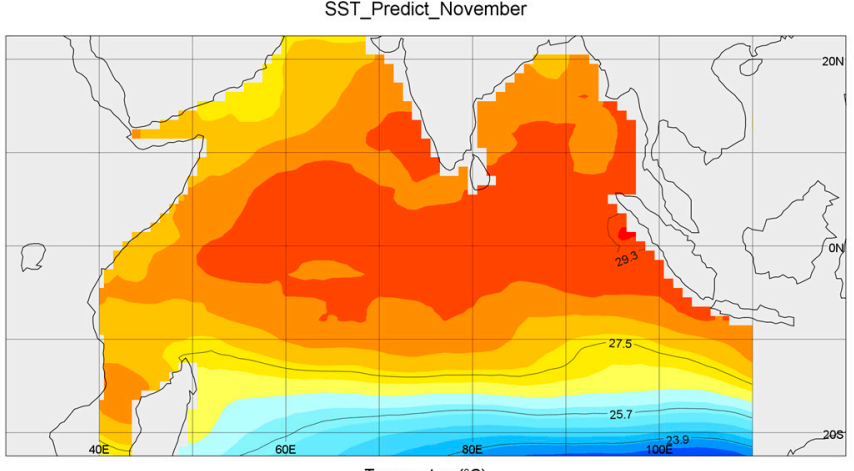

(k)

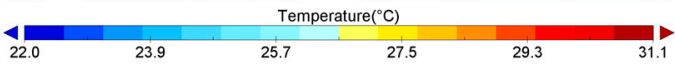

SST_Predict_December

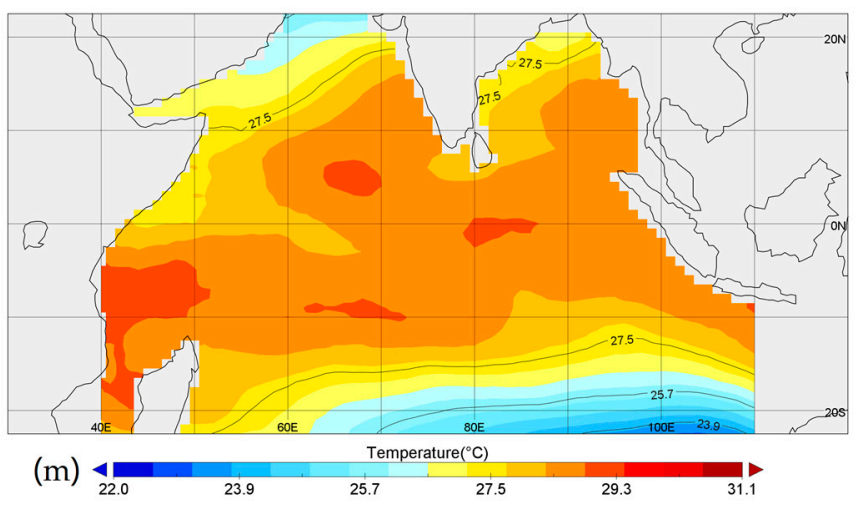

SST_Real_November

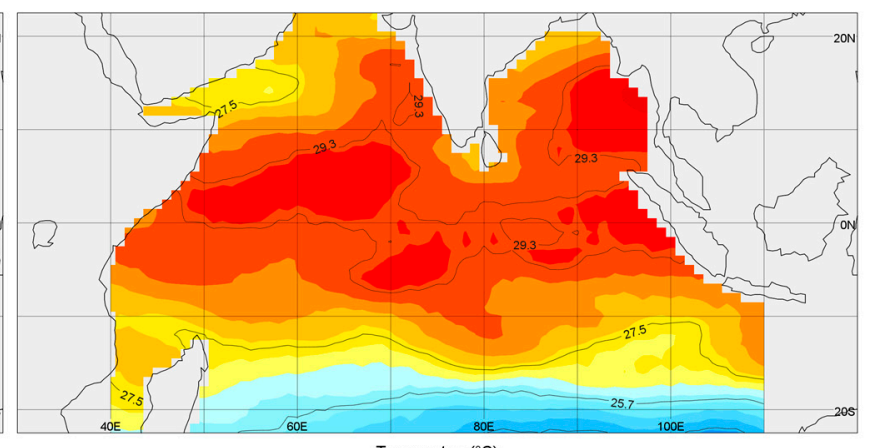

(1)

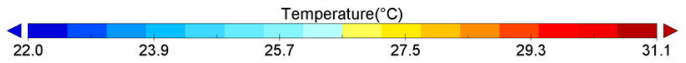

SST_Real_December

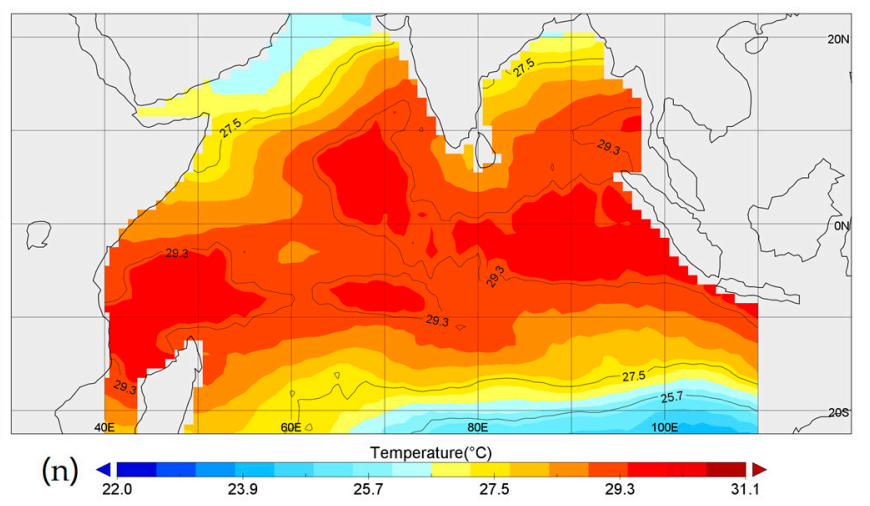

Figure 6. The heat map of the convLSTM model from June 2015 to December 2015 with wind field data and real heat map. (a,c,e,g,i, $, \mathbf{k}, \mathbf{m})$ respectively show the sea surface temperature predicted by the convLSTM model with wind field information, and $(\mathbf{b}, \mathbf{d}, \mathbf{f}, \mathbf{h}, \mathbf{j}, \mathbf{l}, \mathbf{n})$ respectively show the real sea surface temperature from June to December.

\section{Discussion}

This paper focuses on the impact of wind field signal on the prediction accuracy of the IOD index. By adding the wind field signal in each time step, the correlation coefficient of the IOD index from 2015 to 2017 is improved. This paper verifies the prior knowledge of marine physics. The IOD index is affected by the wind field signal, and the average correlation between 2015 and 2018 increased from $81.48 \%$ to $82.84 \%$. In this paper, the existing marine knowledge is verified by the combination of marine physics knowledge and in-depth learning. The model error used in this paper is small. In the prediction of sea surface temperature, the average RMSE from 2015 to 2018 is $0.5493,0.5599,0.462$, and 0.4836 . The average RMSE was $0.5124,0.5532,0.5121$, and 0.4902 after adding the wind field, and the RMSE decreased significantly. After adding the wind field, the average RMSE is $0.5124,0.5532,0.5121$, and 0.4902 . The RMSE decreases significantly, indicating that the local wind field also has an important impact on sea surface temperature.

As a large-scale marine physical phenomenon, IOD is an important factor affecting the seasonal to interannual change of global climate. It is affected by various factors, such as wind field, atmosphere, underwater heat transport, ocean current, and so on. In this paper, the IOD index is predicted by multi-source and multi-modal data concerning the ocean atmosphere. The correlation coefficients from 2015 to 2018 years are $91.51 \%$, $69.50 \%, 69.17 \%$, and $95.73 \%$. It is especially accurate to predict the super IOD events in 15 years and 18 years. In the past, processing complex data requires a lot of manpower and computing resources. Using convLSTM to process multi-dimensional and long-term marine information makes it faster and more accurate. The fitting degree of the fgoals-f2 intra seasonal climate prediction system of the Institute of Atmospheric Physics, Chinese Academy of Sciences for the prediction five months in advance is 0.56 . With the advance 
of the prediction period, the accuracy decreases. The model in this paper predicts seven months in advance, and the gap between IOD index and real value is generally small.

This paper presents a method of adding past six-month wind field information based on the original data to verify whether the wind field has an impact on the IOD prediction. It is very difficult to select the factors that affect physical phenomena in the complex and huge marine data only by manpower. In this paper, deep learning is applied to the research of physical oceanography, which provides support for multi-disciplinary integration. After adding the wind field, the correlation coefficient increases from 2015 to 2017 and decreases in 2018. The improvement of wind field information for IOD anomaly years is relatively obvious, which is consistent with the fact that the wind field signal can guide the deep learning model to better predict the anomalous changes of SST.

This paper forecasts the next seven months through 125 months and completes the processing of ultra-long-time information. However, due to the limited data obtained, it only forecasts the next seven months. In future work, a longer prediction will be completed by adding data. The prediction accuracy of 2016 and 2017 needs to be improved, and the prediction accuracy of 2015 and 2018 is relatively high, which may be due to these years including a super IOD event, negative IOD year, or ordinary years. In future work, we will focus on starting from the prediction of simple features, gradually increasing features, validating the influencing factors that may cause SST anomalies, improving prediction accuracy, and looking for and exploring the key factors affecting the IOD index. Instead of directly predicting the IOD index, this thesis first predicts the sea surface temperature and focuses on exploring the various factors causing the IOD, and the wind field signal is verified. Since the predicted value of the model in this paper is sea surface temperature, the predicted value already contains errors. Recalculating the difference of regional sea surface temperature through the predicted value may increase the error. If the predicted value of sea surface temperature in the eastern Indian Ocean is lower than the real value and the predicted value of sea surface temperature in the western Indian Ocean is higher than the real value, the difference error between the western region and the eastern region will be greater. As a result, the error between the calculated IOD index and the real IOD index is greater.

\section{Conclusions}

In this paper, the convLSTM neural network structure is used to predict the IOD index from June to December and seven months in advance through 125 months of marine atmospheric data. For the selection of features, this paper considers the complex characteristics of the marine atmosphere dataset, including sea surface temperature, underwater temperature, underwater velocity, atmospheric temperature, humidity, and so on. Before inputting the model, combined with the knowledge of physical oceanography, the data are sorted into four-dimensional data. Using the deep learning method to analyze and learn these complex data, we believe that the wind field six months ago will affect the SST anomaly, and the wind field information is added to the characteristics as a factor for predicting the IOD index. The results show that deep learning can be based on existing human knowledge, not just complete black box operation. We use the correlation coefficient to express the fitting degree between our predicted IOD index curve and the real curve. We tested it from 2015 to 2018. The test results show that the correlation coefficient with wind field characteristics reaches $82.84 \%$, and the convLSTM model performs better than CFSv2 model. We believe that deep learning can be optimized by combining the existing professional knowledge of human beings and focusing on learning to achieve better results. However, when we predict super positive and negative IOD, the prediction accuracy is still not good enough, which is where we need to further improve. We hope that the next step will involve prediction based on human prior knowledge to achieve better accuracy and design a more flexible deep learning model. 


\begin{abstract}
Author Contributions: Conceptualization, C.L. and Y.F.; methodology, C.L.; software, C.L.; validation, T.S. and Y.F.; formal analysis, Y.F.; investigation, Y.F.; resources, X.Z.; data curation, T.S.; writing - original draft preparation, C.L.; writing—review and editing, C.L., Y.F. and X.Z.; visualization, C.L.; supervision, Y.F.; project administration, Y.F.; funding acquisition, Y.F. All authors have read and agreed to the published version of the manuscript.
\end{abstract}

Funding: This work was supported in part by the National Research and Development Program of China under Grant 2016YFC1401900 and 2020YFB1710401, and in part by the National Natural Science Foundation of China under Grant 61902367 and Grant 41976185.

Data Availability Statement: Omega: https://psl.noaa.gov/cgi-bin/db_search/DBListFiles.pl? did=198\&tid=96029\&vid=664. Air temperature: https: / / downloads.psl.noaa.gov /Datasets/ncep . reanalysis.derived/surface/air.mon.mean.nc. Uwind: https://downloads.psl.noaa.gov/Datasets / ncep.reanalysis.derived/surface/uwnd.mon.mean.nc. Vwind: https://downloads.psl.noaa.gov/ Datasets/ncep.reanalysis.derived/surface/vwnd.mon.mean.nc. Geopotential height: https://psl. noaa.gov /cgi-bin/db_search/DBSearch.pl? Variable=Geopotential+Height\&group=0\&submit=Search . Specific Humidity: ftp:/ /ftp2.psl.noaa.gov/Datasets/ncep.reanalysis.derived/pressure/shum.mon. mean.nc. Potential temperature: https://psl.noaa.gov/cgi-bin/db_search/DBListFiles.pl?did=98 \&tid=91102\&vid=1913. Salinity: https: / / psl.noaa.gov / cgi-bin/db_search/DBListFiles.pl?did=98 \&tid=91102\&vid=1914. V current: https:/ / psl.noaa.gov/cgi-bin/db_search/DBListFiles.pl?did=98 \&tid=91102\&vid=1920. U current: https:/ / psl.noaa.gov/cgi-bin/db_search/DBListFiles.pl?did=98 \&tid=91102\&vid=1918. Sea surface height: https:/ / psl.noaa.gov/cgi-bin/db_search/DBListFiles. pl?did=98\&tid=91102\&vid=1916. SST: ftp: / / ftp2.psl.noaa.gov/Datasets /COBE2/sst.mon.mean.nc. All accessed on 22 December 2021.

Conflicts of Interest: The authors declare no conflict of interest.

\title{
References
}

1. Saji, N.H.; Goswami, B.N.; Vinayachandran, P.N.; Yamagata, T. A dipole mode in the tropical Indian Ocean. Nature 1999, 401, 360-363. [CrossRef]

2. Xiao, Y.; Zhang, Z.; He, J. Review on research progress of Indian Ocean Dipole. J. Trop. Meteorol. 2009, 25, 621-627.

3. Wang, H.; Kumar, A.; Murtugudde, R.; Narapusetty, B.; Seip, K.L. Covariations between the Indian Ocean dipole and ENSO: A modeling study. Clim. Dyn. 2019, 53, 5743-5761. [CrossRef]

4. Saji, N.H.; Yamagata, T. Possible impacts of Indian Ocean Dipole mode events on global climate. Clim. Res. 2003, 25, 151-169. [CrossRef]

5. Ashok, K.; Guan, Z.; Saji, N.H.; Yamagata, T. Individual and combined infulences of ENSO and the Indian Ocean Dipole on the Indian summer monsoon. J. Clim. 2004, 17, 3141-3155. [CrossRef]

6. Ashok, K.; Guan, Z.; Yamagata, T. Impact of the Indian Ocean Dipole on the relationship between the Indian monsoon rainfall and ENSO. Geophys. Res. Lett. 2001, 28, 4499-4502. [CrossRef]

7. Behera, S.K.; Luo, J.; Masson, S.; Delecluse, P.; Gualdi, S.; Navarra, A.; Yamagata, T. Impact of the Indian Ocean Dipole on the East African short rains: A CGCM study. Clivar Exch. 2003, 27, 43-45. [CrossRef]

8. Chan, S.C.; Behera, S.K.; Yamagata, T. Indian Ocean Dipole influence on South American rainfall. Geophys. Res. Lett. 2008, 35, L14S12. [CrossRef]

9. Guan, Z.; Yamagata, T. The unusual summer of 1994 in East Asia: IOD teleconnections. Geophys. Res. Lett. 2003, 30, 235-250. [CrossRef]

10. Qiu, Y.; Cai, W.; Guo, X.; Ng, B. The asymmetric influence of the positive and negative IOD events on China's rainfall. Sci. Rep. 2014, 4, 4943. [CrossRef]

11. Xiao, M.; Zhang, Q.; Singh, V.P. Influences of ENSO, NAO, IOD and PDO on seasonal precipitation regimes in the Yangtze River basin, China. Int. J. Climatol. 2015, 35, 3556-3567. [CrossRef]

12. Xu, K.; Zhu, C.; Wang, W. The cooperative impacts of the El Niño-Southern Oscillation and the Indian Ocean Dipole on the interannual variability of autumn rainfall in China. Int. J. Climatol. 2016, 36, 1987-1999. [CrossRef]

13. Wajsowicz, R.C. Climate variability over the tropical Indian Ocean sector in the NSIPP seasonal forecast system. J. Clim. 2004, 17, 4783-4804. [CrossRef]

14. Wajsowicz, R.C. Seasonal-to-interannual forecasting of tropical Indian Ocean sea surface temperature anomalies: Potential predictability and barriers. J. Clim. 2007, 20, 3320-3343. [CrossRef]

15. Luo, J.; Masson, S.; Behera, S.; Yamagata, T. Experimental forecasts of the Indian Ocean Dipole using a coupled OAGCM. J. Clim. 2007, 20, 2178-2190. [CrossRef]

16. Zhao, M.; Hendon, H. Representation and prediction of the Indian Ocean Dipole in the POAMA seasonal forecast model. Q. J. R. Meteorol. Soc. 2009, 135, 337-352. [CrossRef] 
17. Bao, Q.; Wu, X.; Li, J.; Wang, L.; He, B.; Wang, X.; Liu, Y.; Wu, G. Prediction of El Nino and Indian Ocean Dipole in autumn and winter 2018-2019. Sci. Bull. 2019, 64, 73-78.

18. Hu, S.; Wu, B.; Zhou, T. The reward technique of the recent climate prediction system iap-decpres for the Indian Ocean Dipole: A comparison between full field assimilation and anomaly field assimilation. Atmos. Sci. 2019, 43, 831-845.

19. Zhao, S.; Jin, F.-F.; Stuecker, M.F. Improved Predictability of the Indian Ocean Dipole Using Seasonally Modulated ENSO Forcing Forecasts. Geophys. Res. Lett. 2019, 46, 9980-9990. [CrossRef]

20. Ham, Y.G.; Kim, J.H.; Luo, J.J. Deep learning for multi-year ENSO forecasts. Nature 2019, 573, 568-572. [CrossRef]

21. Sarkar, P.P.; Janardhan, P.; Roy, P. A novel deep neural network model approach to predict Indian Ocean dipole and Equatorial Indian Ocean oscillation indices. Dyn. Atmos. Oceans 2021, 96, 101266. [CrossRef]

22. Lawrence, S.; Giles, C.L.; Tsoi, A.C.; Back, A.D. Face recognition: A convolutional neural-network approach. IEEE Trans. Neural Netw. 1997, 8, 98-113. [CrossRef] [PubMed]

23. Swapna, A. Machine learning based sample extraction for automatic speech recognition using dialectal Assamese speech. Neural Netw. 2016, 78, 97-111.

24. Xiong, J.; Bi, R.; Tian, Y.; Liu, X.; Wu, D. Towards Lightweight, Privacy-Preserving Cooperative Object Classification for Connected Autonomous Vehicles. IEEE Internet Things J. 2021, 1. [CrossRef]

25. Xiong, J.; Bi, R.; Zhao, M.; Guo, J.; Yang, Q. Edge-Assisted Privacy-Preserving Raw Data Sharing Framework for Connected Autonomous Vehicles. IEEE Wirel. Commun. 2020, 27, 24-30. [CrossRef]

26. Bi, R.; Chen, Q.; Xiong, J.; Liu, X. Design method of secure computing protocol for deep neural network. Chin. J. Netw. Inf. Secur. 2020, 6, 130-139.

27. Zhang, Q.; Wang, H.; Dong, J.; Zhong, G.; Sun, X. Prediction of Sea Surface Temperature Using Long Short-Term Memory. IEEE Geosci. Remote Sens. Lett. 2017, 14, 1745-1749. [CrossRef]

28. Krizhevsky, A.; Sutskever, I.; Hinton, G.E. ImageNet classification with deep convolutional neural networks. Commun. ACM 2017, 60, 84-90. [CrossRef]

29. Shi, X.; Chen, Z.; Wang, H.; Yeung, D.-Y.; Wong, W.-K.; Woo, W. Convolutional LSTM Network: A Machine Learning Approach for Precipitation Nowcasting. arXiv 2015, arXiv:1506.04214. 